\title{
Splints of classical root systems
}

\author{
David A. Richter \\ Department of Mathematics, MS 5248 \\ Western Michigan University \\ Kalamazoo MI 49008-5248 \\ david.richter@wmich.edu
}

\begin{abstract}
This article introduces a new term "splint" and classifies the splints of the classical root systems. The motivation comes from representation theory of semisimple Lie algebras. In a few instances, splints play a role in determining branching rules of a module over a complex semisimple Lie algebra when restricted to a subalgebra. In these particular cases, the set of submodules with respect to the subalgebra themselves may be regarded as the character of another Lie algebra.
\end{abstract}

Keywords: branching rule; root system; splint

AMS Mathematics Subject Classification (2000): 17B05, 17B10.

\section{Introduction}

This paper is concerned with a branching rule for a pair $\mathfrak{g}_{1} \subset \mathfrak{g}$ of semisimple Lie algebras in which the highest weights of a representation $V$ with respect to $\mathfrak{g}_{1}$ themselves may serve as the weights of another semisimple Lie algebra. The particular phenomenon has been mentioned in the literature only a few times, and generally only parenthetically; see [2] and [5].

It is perhaps easiest to see the phenomenon the case where $\mathfrak{g}$ is the 14-dimensional exceptional simple Lie algebra $\mathfrak{g}_{2}$ and the subalgebra is $\mathfrak{s l}_{3}$. If $V$ is any finite-dimensional irreducible module for $\mathfrak{g}_{2}$, then the branching into irreducible representations of $\mathfrak{s l}_{3}$ has the pattern of a weight diagram for $\mathfrak{s l}_{3}$. As [2] explains, this follows from the fact that the difference between the root systems of $\mathfrak{g}_{2}$ and $\mathfrak{s l}_{3}$ is a copy of the root system for $\mathfrak{s l}_{3}$.

A less trivial example occurs when $\mathfrak{g}=\mathfrak{b}_{2} \cong \mathfrak{s o}_{5}$. Recall that the set

$$
\Delta^{+}=\left\{e_{1}, e_{2}, e_{1}-e_{2}, e_{1}+e_{2}\right\}
$$

serves as a system of positive roots for this Lie algebra, where $\left\{e_{1}, e_{2}\right\}$ is an orthonormal basis for the dual of a prescribed Cartan subalgebra. Choose $\mathfrak{g}_{1}=\mathfrak{a}_{1} \cong \mathfrak{s l}_{2}$ as the Lie subalgebra corresponding to the subset

$$
\Delta_{1}^{+}=\left\{e_{1}-e_{2}\right\} .
$$

Next, let $V$ be a finite-dimensional irreducible module over $\mathfrak{g}$. Then $V$ is a module over $\mathfrak{g}_{1}$ and one may write

$$
V=\bigoplus_{\mu} V_{\mu},
$$


where each $V_{\mu}$ is an irreducible $\mathfrak{g}_{1}$-module with highest weight $\mu$. Then, as the reader may check, the weights $\mu$ appearing in this summand have the pattern of a weight diagram of a representation of $\mathfrak{s l}_{3}$. This occurs despite the fact that $\mathfrak{a}_{2}$ is not a subalgebra of $\mathfrak{b}_{2}$.

These examples motivate the introduction of the term "splint". Roughly, a splint of a root system $\Delta$ is a partition $\Delta_{1} \cup \Delta_{2}$ into two subsets, each of which have the additive, but not necessarily metrical, properties of a root system. The aim in this study is to classify all splints of root systems for complex semisimple Lie algebras. This problem is slightly more general than what is needed with regards to branching rules. In the example above, one of the "components" $\Delta_{1}$ corresponds to a Lie subalgebra of $\mathfrak{g}$, but one will not find this constraint in the definitions; there are few enough cases to sort through if the reader wishes to find these.

One of the ingredients of the term "splint" is the idea of a root system embedding. The definition given here is novel, although [1] introduces a similar definition. The reference [6] is given because the techniques used there seem the closest to the ideas expressed in this introduction.

\section{Definitions}

The purpose of this section is is to define what it means for a root system to splinter, but one first needs some preliminary terminology. First of all, the term "simple root system" refers to one appearing in Cartan's list

$$
A_{r}(r \geq 1), B_{r}(r \geq 2), C_{r}(r \geq 3), D_{r}(r \geq 4), E_{6}, E_{7}, E_{8}, F_{4}, G_{2} \text {, }
$$

corresponding to four classical sequences of and five exceptional complex simple Lie algebras, [3, 4.

If $\mathfrak{g}$ is complex semisimple, then its root system is a disjoint union of various copies of these, each corresponding to a summand of $\mathfrak{g}$ into simple components. One may abstract this as follows: Suppose $\Delta_{1}$ and $\Delta_{2}$ are root systems. Then one assumes already that $\Delta_{1}$ and $\Delta_{2}$ are subsets of vector spaces $V_{1}$ and $V_{2}$ respectively. The "direct sum" of the root systems is then

$$
\Delta_{1}+\Delta_{2}=\left\{(\alpha, 0): \alpha \in \Delta_{1}\right\} \cup\left\{(0, \alpha): \alpha \in \Delta_{2}\right\} \subset V_{1} \oplus V_{2} .
$$

The direct sum operation has the following features: First, if $\Delta=\Delta_{1}+\Delta_{2}$ with $\alpha_{1} \in \Delta_{1}$ and $\alpha_{2} \in \Delta_{2}$, then $\alpha_{1}+\alpha_{2} \notin \Delta$. Second, if there exist roots $\alpha_{1}, \alpha_{2} \in \Delta$ such that $\alpha_{1}+\alpha_{2}$ is also a root, then the root system cannot be written as a direct sum $\Delta=\Delta_{1}+\Delta_{2}$ where $\alpha_{1} \in \Delta_{1}$ and $\alpha_{2} \in \Delta_{2}$. Third, if one may write $\Delta=\Delta_{1} \cup \Delta_{2}$ as a disjoint union where for all $\alpha_{1} \in \Delta_{1}$ and all $\alpha_{2} \in \Delta_{2}$, the vector $\alpha_{1}+\alpha_{2}$ is not a root, then $\Delta$ is the direct sum of $\Delta_{1}$ and $\Delta_{2}$.

Definition 2.1 Suppose $\Delta_{0}$ and $\Delta$ are root systems. Then $\iota$ is an "embedding" if (a) it is a 1-1 function from $\Delta_{0}$ to $\Delta$, and (b)

$$
\iota(\gamma)=\iota(\alpha)+\iota(\beta)
$$


for all $\alpha, \beta, \gamma \in \Delta_{0}$ such that $\gamma=\alpha+\beta$.

It is convenient to write " $\Delta_{0} \hookrightarrow \Delta$ " in order to imply that there is an embedding from $\Delta_{0}$ to $\Delta$ and to say, under these circumstances, that $\Delta_{0}$ "embeds" in $\Delta$. Given this definition, one may summarize some of the preceeding observations:

Proposition 2.2 Suppose $\Delta$ is a root system. Then at least one simple component of $\Delta$ is not isomorphic to $A_{1}$ if and only if there is an embedding $A_{2} \hookrightarrow \Delta$.

The following tells what it means for a root system to splinter.

Definition 2.3 A root system $\Delta$ "splinters" as $\left(\Delta_{1}, \Delta_{2}\right)$ if there are two embeddings $\iota_{1}$ : $\Delta_{1} \hookrightarrow \Delta$ and $\iota_{2}: \Delta_{2} \hookrightarrow \Delta$ where (a) $\Delta$ is the disjoint union of the images of $\iota_{1}$ and $\iota_{2}$ and (b) neither the rank of $\Delta_{1}$ nor the rank of $\Delta_{2}$ exceeds the rank of $\Delta$

It is equivalent to say that $\left(\Delta_{1}, \Delta_{2}\right)$ is a "splint" of $\Delta$. Each component $\Delta_{1}$ and $\Delta_{2}$ is a "stem" of the splint $\left(\Delta_{1}, \Delta_{2}\right)$.

Thus far, these definitions do not mention inner products. As explained earlier, since the factorization of the Weyl denominator does not require an inner product, the focus here is on the additive properties of root systems. Nevertheless, as defined in many places, for example as in [3, a root system is a particular type of subset of a vector space equipped with a Euclidean inner product. In this regard, it is useful to distinguish between "metric root systems," which are endowed with an inner product and mere "root systems", described above.

It is also useful to introduce some terminology relating metric root systems with general root systems. Suppose $\iota: \Delta_{0} \hookrightarrow \Delta$ is an embedding, and suppose that the inner products associated to $\Delta_{0}$ and $\Delta$, respectively, are $\langle,\rangle_{0}$ and $\langle$,$\rangle . Call the embedding \iota$ "metric" if there is a positive scalar $\lambda$ such that

$$
\langle\alpha, \beta\rangle_{0}=\lambda\langle\iota(\alpha), \iota(\beta)\rangle
$$

for all $\alpha, \beta \in \Delta_{0}$ and "non-metric" otherwise. An imbedding $\iota: \Delta_{0} \hookrightarrow \Delta$ may not be metric even if the restriction of $\iota$ to every simple component is metric; call such a non-metric embedding "semimetric".

The structure of a root system is characterized by the additive properties of its corresponding system of positive roots. Thus, if $\Delta$ is the set of all roots, positive and negative, and $\Delta_{1}^{+}$and $\Delta_{2}^{+}$are two choices of positive roots, then there is a bijection $f: \Delta_{1}^{+} \rightarrow \Delta_{2}^{+}$ having the property that $f(\alpha+\beta)$ is a root if and only if $\alpha+\beta$ is a root. Hence, it is necessary only to consider positive root systems. This should be considered as the 0th definition of this section: A "simple root system" is any system of positive roots for one of the root systems listed above, and, more generally, a "root system" is any direct sum of these.

In the sequel, it is necessary to be familiar with some presentations of simple root systems. Set

$$
A_{r}=\left\{e_{i}-e_{j}: 0 \leq i<j \leq r\right\}, D_{r}=\left\{e_{i} \pm e_{j}: 1 \leq i<j \leq r\right\},
$$




$$
B_{r}=D_{r} \cup\left\{e_{i}: 1 \leq i \leq r\right\} \text {, and } C_{r}=D_{r} \cup\left\{2 e_{i}: 1 \leq i \leq r\right\},
$$

where $\left\{e_{i}: i \in I\right\}$ is assumed to be orthonormal for any set $I$. Notice that every root of $A_{r}$ and every root of $D_{r}$ has norm 2 . None of the well-known presentations of $E_{6}, E_{7}$, and $E_{8}$ are required here, but one does need the convention that every element of any of these root system has norm 2. Finally,

$$
F_{4}=\left\{2 e_{i}: 1 \leq i \leq 4\right\} \cup\left\{e_{1} \pm e_{2} \pm e_{3} \pm e_{4}\right\} \cup\left\{e_{i} \pm e_{j}: 1 \leq i<j \leq 4\right\}
$$

and

$$
G_{2}=\left\{e_{1}-e_{2}, e_{2}-e_{3}, e_{1}-e_{3}, 2 e_{1}-e_{2}-e_{3}, e_{1}+e_{2}-2 e_{3}, e_{1}-2 e_{2}+e_{3}\right\}
$$

\section{Results}

The main result is the following:

Theorem 3.1 The table of splints of simple root systems given in the appendix is complete.

In order to establish this, one first needs some moderately general results. First, it should be clear that the most difficult part of the problem involves splintering the simple root systems. Indeed, if a root system is a direct sum $\Delta=\Delta_{1}+\Delta_{2}$, then obviously $\left(\Delta_{1}, \Delta_{2}\right)$ is a splint of $\Delta$; one may regard this as a trivial splint. The next result is straighforward, provided one uses the axioms for root systems developed, for example, in [3]:

Proposition 3.2 Suppose $\iota_{1}: \Delta_{1} \hookrightarrow \Delta$ and $\iota_{2}: \Delta_{2} \hookrightarrow \Delta$ are metric embeddings. Then, if it is non-empty, the intersection of the images of $\iota_{1}$ and $\iota_{2}$ is a root system metrically embedded in $\Delta$.

Next, it is useful to characterize root systems of type $A D E$ in this context:

Proposition 3.3 Let $\Delta$ be a simple root system. Then $B_{2} \hookrightarrow \Delta$ if and only if $\Delta$ has type $B C F G$.

\section{ProOF.}

Notice that $B_{2}$ has roots $\alpha$ and $\beta$ such that both $\alpha+\beta$ and $2 \alpha+\beta$ are also roots, as does every root system of type $B C F G$. On the other hand, no root sytem of type $A D E$ has such a pair of roots.

Corollary 3.4 Suppose $\Delta_{0}$ is simple and $\Delta$ has type ADE. If $\iota: \Delta_{0} \hookrightarrow \Delta$ is an embedding, then $\Delta_{0}$ has type $A D E$ and $\iota$ is metric. 


\section{PROOF.}

First use the characterization of root systems of type $A D E$ given above; since $\Delta_{0}$ is simple, this implies that $\Delta_{0}$ has type $A D E$. Next, assuming all roots have norm 2 , if $\alpha$ and $\beta$ are roots, then $\alpha+\beta$ is a root if and only if $\langle\alpha, \beta\rangle=-1$. This shows that $\iota$ is metric.

One may now proceed with the various cases. There are two sections for handling the special cases, divided according to whether the simple root system has type $A D E$ or $B C F G$. For each of the infinite sequences $A_{r}, B_{r}, C_{r}$, and $D_{r}$, there are two different parts to classifying the splints. First one must determine all splints when $r$ is small, and then one must show that when $r$ is sufficiently large, the number of splints is severely limited.

\subsection{Root sytems of type $A D E$}

$A_{r}$

Since it has only 1 element, it is obvious that that $A_{1}$ does not splinter. Given any $r \geq 3$, there are at least two splints of $A_{r}$, namely $\left(r A_{1}, A_{r-1}\right)$ and $\left(A_{1}+A_{r-1},(r-1) A_{1}\right)$. One may describe the first splint by writing

$$
\Delta_{1}=\left\{e_{i}-e_{r}: 0 \leq i \leq r-1\right\} \text { and } \Delta_{2}=\left\{e_{i}-e_{j}: 0 \leq i<j \leq r-1\right\},
$$

and the second by

$$
\Delta_{1}=\left\{e_{i}-e_{j}: 0 \leq i<j \leq r-1\right\} \cup\left\{e_{0}-e_{r}\right\} \text { and } \Delta_{2}=\left\{e_{i}-e_{r}: 1 \leq i \leq r-1\right\} .
$$

Evidently the two splints are closely related, one being obtained from the other by "shifting" $e_{0}-e_{r}$ between the two summands. These splints coincide when $r=2$, yielding the splint $\left(A_{1}, 2 A_{1}\right)$ of $A_{2}$. The root system $A_{3}$ has an additional splint as $\left(3 A_{1}, 3 A_{1}\right)$, letting

$$
\Delta_{1}=\left\{e_{0}-e_{1}, e_{1}-e_{2}, e_{0}-e_{3}\right\} \text { and } \Delta_{2}=\left\{e_{2}-e_{3}, e_{0}-e_{2}, e_{1}-e_{3}\right\} .
$$

Notice that in each of these subsets, there is a pair of orthogonal roots. Thus, it seems appropriate to denote this splint by $\left(A_{1}+D_{2}, A_{1}+D_{2}\right)$. The root system $A_{4}$ has two additional splints as $\left(A_{2}+2 A_{1}, A_{2}+2 A_{1}\right)$ and $\left(2 A_{2}, 4 A_{1}\right)$. One may describe the first by letting

$$
\Delta_{1}=\left\{e_{2}-e_{3}, e_{3}-e_{4}, e_{2}-e_{4}, e_{0}-e_{3}, e_{1}-e_{4}\right\}
$$

and

$$
\Delta_{2}=\left\{e_{0}-e_{1}, e_{1}-e_{2}, e_{0}-e_{2}, e_{1}-e_{3}, e_{0}-e_{4}\right\}
$$

and the second by

$$
\Delta_{1}=\left\{e_{0}-e_{1}, e_{1}-e_{2}, e_{0}-e_{2}, e_{2}-e_{3}, e_{3}-e_{4}, e_{2}-e_{4}\right\}
$$

and

$$
\Delta_{2}=\left\{e_{1}-e_{3}, e_{0}-e_{3}, e_{1}-e_{4}, e_{0}-e_{4}\right\} .
$$

Again, due to the presence of pairs of orthogonal roots under the usual inner product, these splints may be denoted $\left(A_{2}+D_{2}, A_{2}+D_{2}\right)$ and $\left(2 A_{2}, 2 D_{2}\right)$ respectively. 
Lemma 3.5 If $\Delta$ is simple and $\Delta \hookrightarrow A_{r}$, then $\Delta \cong A_{s}$ for some $s \leq r$.

\section{Proof.}

If one writes the highest root of $A_{r}$ as a linear combination of simple roots, then every coefficient is equal to 1 .

Proposition 3.6 (a) The root system $A_{2}$ has exactly one splint. (b) The root system $A_{3}$ has exactly three splints. (c) The root system $A_{4}$ has exactly four splints. (d) The root system $A_{5}$ has exactly two splints.

\section{ProOF.}

Parts (a) and (b) are evident. Parts (c) and (d) follow quickly after observing the following: If $A_{s} \hookrightarrow A_{r}$ and $A_{t} \hookrightarrow A_{r}$ are embeddings with disjoint images, then $s+t \leq r$. Suppose $r=4$. It is clear that there are only two splints having $A_{3}$ as one of the components. If one of the components is $A_{2}$, then every other component is isomorphic to either $A_{2}$ or $A_{1}$. By simply listing the cases, one can eliminate all but the two described above. The argument for $A_{5}$ is similar.

Lemma 3.7 Suppose $r \geq 3$ and $s \geq 2$. If $A_{r}$ has a splint where $A_{s}$ is a component, then $A_{r-1}$ has a splint having $A_{s-1}$ as a component.

\section{ProOF.}

Suppose $\left(\Delta_{1}, \Delta_{2}\right)$ is a splint of $A_{r}$ with having $\iota: A_{s} \hookrightarrow \Delta_{1}$ as a component with $s \geq 2$. Without loss of generality, one may assume that the roots in the image of $\iota$ have the form $e_{i}-e_{j}$, where $i<j$ and $i, j \in\{0,1,2, \ldots, s-1, r\}$. Consider restricting the splint to the embedding $\kappa: A_{r-1} \hookrightarrow A_{r}$ where the image of $\kappa$ consists of roots of the form $e_{i}-e_{j}$ where $0 \leq i<j \leq r-1$. Since $\kappa$ is metric and all components of $\left(\Delta_{1}, \Delta_{2}\right)$ are embedded metrically, this yields a splint of $A_{r-1}$ having $A_{s-1}$ as a component.

Proposition 3.8 Suppose $r \geq 6$. If $\left(\Delta_{1}, \Delta_{2}\right)$ is a splint of $A_{r}$ having $A_{s}$ as a component, then $s \in\{1, r-1\}$.

\section{ProOF.}

If $r \geq 6$, then one may argue by contradiction, using the preceeding results. 
Combining the results of this section, one sees that $A_{r}$ has exactly two splints when $r \geq 5$.

$D_{r}$

There is only one value of $r \geq 4$ for which $D_{r}$ splinters, namely $r=4$. In this case there is only one splint $\left(2 A_{2}, 2 A_{2}\right)$, and one may present this by writing

$$
\Delta_{1}=\left\{e_{1}-e_{2}, e_{2}-e_{4}, e_{1}-e_{4}\right\} \cup\left\{e_{3}+e_{4}, e_{1}-e_{3}, e_{1}+e_{4}\right\}
$$

and

$$
\Delta_{2}=\left\{e_{3}-e_{4}, e_{2}+e_{4}, e_{2}+e_{3}\right\} \cup\left\{e_{2}-e_{3}, e_{1}+e_{3}, e_{1}+e_{2}\right\} .
$$

Lemma 3.9 The root system $D_{5}$ does not splinter.

\section{Proof.}

Suppose $\left(\Delta_{1}, \Delta_{2}\right)$ is a splint of $D_{5}$. Then one has $\left|\Delta_{1}\right|+\left|\Delta_{2}\right|=\left|D_{5}\right|=20$, so, without loss of generality, one may assume that $\left|\Delta_{1}\right| \geq 10$. Also, from an earlier proposition, every component of $\Delta_{1}$ is embedded metrically. There are exactly five root systems with rank not exceeding 5 which have these properties, namely $A_{4}, A_{1}+A_{4}, D_{4}, A_{1}+D_{4}$, and $A_{5}$. One must rule out all five cases.

Suppose $A_{5}$ is a component. Then there is a metric embedding $A_{4} \hookrightarrow A_{5}$, and one may assume without loss of generality that the roots in the image of $A_{4}$ are those of the form $e_{i}-e_{j}$ such that $1 \leq i<j \leq 5$. In particular, the simple roots are $e_{i}-e_{i+1}$ for $1 \leq i \leq 4$. Every root of $D_{5}$ not lying in this image of $A_{4}$ then has the form $e_{i}+e_{j}$. Since $A_{5}$ is a component, there is a root of the form $e_{i}+e_{j}$ such that

$$
\left\{e_{i}-e_{i+1}: 1 \leq i \leq 4\right\} \cup\left\{e_{i}+e_{j}\right\}
$$

is a system of simple roots. This is impossible, so $A_{5}$ cannot be a component.

Next, suppose $A_{4}$ is a component. Then the embedding of $A_{4}$ is metric and, as above, one may assume that the image consists of all roots of the form $e_{i}-e_{j}$, where $1 \leq i<j \leq 5$. Every root not lying in the image then has the form $e_{i}+e_{j}$. Notice that $A_{2}$ hence does not embed in the remaining roots and every other simple component of the splint is isomorphic to $A_{1}$. Since there are 10 roots of this form and $A_{4}$ has rank 4 , the total of the ranks of all the simple components is then 14 . Since this exceeds 10, this violates the definition of "splint".

An argument in the case that $D_{4}$ is a component similarly leads to a contradiction.

Proposition 3.10 If $r \geq 6$, then $D_{r}$ does not splinter.

Proof. 
Suppose $\left(\Delta_{1}, \Delta_{2}\right)$ is a splint of $D_{r}$ where $r \geq 6$. Then every component is embedded metrically. Moreover, there is at least one embedding $D_{5} \hookrightarrow D_{r}$ whose image contains elements of both $\Delta_{1}$ and $\Delta_{2}$. Such an embedding is metric and all the components of the splint are embedded metrically, so such a splint restricts to a splint of $D_{5}$, a contradiction.

\section{$E_{r}$}

Proposition 3.11 None of the root systems $E_{6}, E_{7}$, nor $E_{8}$ splinters.

ProOF.

Since $D_{5}$ embeds metrically in $E_{r}$ for all $r \in\{6,7,8\}$ and $E_{r}$ has type $A D E$, one may use the same argument which was used for the cases of $D_{r}$ with $r \geq 6$.

\subsection{Root sytems of type $B C F G$}

$G_{2}$

By inspection, one may quickly establish:

Proposition 3.12 The root system $G_{2}$ has exactly two splints.

These are $\left(A_{2}, A_{2}\right)$ and $\left(2 A_{1}, B_{2}\right)$. One obtains the former of these by partitioning $G_{2}$ into its long and short roots; thus,

$$
\Delta_{1}=\left\{2 e_{1}-e_{2}-e_{3}, e_{1}-2 e_{2}+e_{3}, e_{1}+e_{2}-2 e_{3}\right\} \text { and } \Delta_{2}=\left\{e_{1}-e_{2}, e_{1}-e_{3}, e_{2}-e_{3}\right\} .
$$

In this splint, both components are embedded metrically. One obtains the latter by considering two of the long roots as copies of $A_{1}$. Thus,

$$
\Delta_{1}=\left\{e_{1}-2 e_{2}+e_{3}, e_{1}+e_{2}-2 e_{3}\right\} \text { and } \Delta_{2}=\left\{2 e_{1}-e_{2}-e_{3}, e_{1}-e_{2}, e_{1}-e_{3}, e_{2}-e_{3}\right\} .
$$

\section{$F_{4}$}

Similar to the case of $G_{2}$, one obtains the totally metric splint $\left(D_{4}, D_{4}\right)$ when one partitions the roots of $F_{4}$ into long and short roots.

Proposition 3.13 The root system $F_{4}$ has exactly one splint. 


\section{Proof.}

One must show that there are no other splints other than that described above. Suppose $\left(\Delta_{1}, \Delta_{2}\right)$ is a splint of $F_{4}$ and consider the possible cardinalities of the components $\Delta_{1}$ and $\Delta_{2}$. One may quickly tabulate the cardinalities of all the root systems, simple or not, with rank not exceeding 4 . One first finds that $D_{4}$ is the only such root system with cardinality 12. This shows that the only way to splinter $D_{4}$ into two root systems each with cardinality 12 is to splinter it as $\left(D_{4}, D_{4}\right)$.

At least one of these splints has already been described, so one must now show that there aren't any others. A tedious check reveals the existence of at least one non-metric embedding of $D_{4} \hookrightarrow F_{4}$. This has simple roots

$$
\Sigma=\left\{e_{1}-e_{4}, e_{2}-e_{4}, e_{3}-e_{4}, 2 e_{4}\right\},
$$

and the image consists of 9 short roots

$$
\left\{e_{1} \pm e_{4}, e_{2} \pm e_{4}, e_{3} \pm e_{4}, e_{1}+e_{2}, e_{1}+e_{3}, e_{2}+e_{3}\right\}
$$

and 3 long roots

$$
\left\{2 e_{4}, e_{1}+e_{2}+e_{3} \pm e_{4}\right\} .
$$

Moreover, again by some tedious computations, one may see that every other embedding $D_{4} \hookrightarrow F_{4}$ which is not one of the two metric embeddings described earlier has precisely 3 long roots and 9 short roots. Thus, the remaining set of roots not lying in the image of this embedding, which contains 3 short roots and 9 long roots, cannot be the image of such an embedding. Therefore, there is only one splint of $F_{4}$ where the cardinality of each component is equal to 12 .

Next, there are only two root systems having rank not exceeding 4 with cardinality between 12 and 24 , namely $B_{4}$ and $C_{4}$, and each of these has cardinality 16 . Without loss of generality, assume $\Delta_{1}$ has cardinality 16 . Then $\Delta_{2}$ must have cardinality 8 , and another inspection of the table reveals that $\Delta_{2}$ must be isomorphic to $2 B_{2}$. Thus, the aim now is to rule out the cases that $\left(\Delta_{1}, \Delta_{2}\right)$ may be isomorphic to either $\left(B_{4}, 2 B_{2}\right)$ or $\left(C_{4}, 2 B_{2}\right)$. In order to rule these out, one observes that an embedding of either $B_{4}$ or $C_{4}$ in $F_{4}$ must be metric. In the former case, $B_{4}$ contains all the long roots of $F_{4}$ and in the latter, $C_{4}$ contains all the short roots. In either case, the balance consists of roots of only one length, and thus cannot contain a copy of $B_{2}$.

\section{$B_{r}$}

For any $r \geq 2$, the root system $B_{r}$ has a splint $\left(D_{r}, r A_{1}\right)$ into long and short roots, given as

$$
\Delta_{1}=\left\{e_{i} \pm e_{j}: 1 \leq i<j \leq r\right\}, \text { and } \Delta_{2}=\left\{e_{i}: 1 \leq i \leq r\right\} .
$$

This splint is distinguished because both $\Delta_{1}$ and $\Delta_{2}$ are embedded metrically. The root system $B_{2}$ has two additional splints. The first, $\left(A_{1}, A_{2}\right)$, may be given by

$$
\Delta_{1}=\left\{e_{1}-e_{2}\right\} \text { and } \Delta_{2}=\left\{e_{1}, e_{2}, e_{1}+e_{2}\right\},
$$


and the second, $\left(2 A_{1}, 2 A_{1}\right)$, may be given by

$$
\Delta_{1}=\left\{e_{1}, e_{1}-e_{2}\right\} \text { and } \Delta_{2}=\left\{e_{2}, e_{1}+e_{2}\right\} .
$$

The root system $B_{3}$ has an additional splint as $\left(3 A_{1}, A_{3}\right)$ given by

$$
\Delta_{1}=\left\{e_{1}+e_{2}, e_{1}+e_{3}, e_{2}+e_{3}\right\} \text { and } \Delta_{2}=\left\{e_{1}-e_{2}, e_{1}-e_{3}, e_{2}-e_{3}, e_{1}, e_{2}, e_{3}\right\} .
$$

There are no other splints of $B_{r}$ besides these. The proof depends mainly on the analysis of the $D_{r}$ root systems and a detailed description of non-metric embeddings $A_{s} \hookrightarrow B_{r}$.

Lemma 3.14 The root system $C_{3}$ does not embed in $B_{r}$ for any $r \geq 2$.

\section{ProOF.}

If $\alpha, \beta$, and $\frac{1}{2}(\alpha+\beta)$ are roots in $B_{r}$, then $\alpha$ and $\beta$ are long and orthogonal to each other, while $\frac{1}{2}(\alpha+\beta)$ is short. Next, $C_{3}$ contains three roots $\alpha, \beta$, and $\gamma$ with the property that the vectors $\frac{1}{2}(\alpha+\beta), \frac{1}{2}(\alpha+\gamma)$, and $\frac{1}{2}(\beta+\gamma)$ are also roots. Suppose such roots lie in $B_{r}$. All of the short roots in $B_{r}$ are orthogonal to each other, and so one has, for instance,

$$
\begin{aligned}
0 & =\langle\alpha+\beta, \alpha+\gamma\rangle \\
& =\langle\alpha, \alpha\rangle+\langle\alpha, \gamma\rangle+\langle\beta, \alpha\rangle+\langle\beta, \gamma\rangle \\
& =2+0+0+0 \\
& =2
\end{aligned}
$$

a contradiction.

Proposition 3.15 If $s \geq 4$, then every embedding $D_{s} \hookrightarrow B_{r}$ is metric.

\section{ProOF.}

This may be considered as a corollary to the analysis of the $F_{4}$ root system. Every nonmetric embedding $D_{4} \hookrightarrow F_{4}$ contains exactly 3 long roots and 9 short roots. Also, there is a metric embedding $B_{4} \hookrightarrow F_{4}$. However, the root system $B_{4}$ has only 4 short roots, so there is no non-metric embedding $D_{4} \hookrightarrow B_{4}$. The cases when $s \geq 5$ follow by restricting to this case.

Lemma 3.16 If a non-surjective embedding $\iota: \Delta \hookrightarrow B_{r}$ contains all the long roots of $B_{r}$, then $\Delta \cong D_{r}$.

\section{ProOF.}

This is obvious if $r=2$. If $r \geq 3$, then $\Delta$ is simple since it has rank $r$ and contains all the roots of $D_{r}$. Since the image of $\iota$ contains all the $r(r-1)$ long roots of $D_{r}$ and not all of 
the short roots of $B_{r}$, there is an integer $k \in\{0,1,2, \ldots, r-1\}$ such that the cardinality of $\Delta$ is $r(r-1)+k$. After an examination of the cardinalities of all the simple root systems, one concludes that $k=0$.

Despite the fact that an embedding $\iota: A_{s} \hookrightarrow B_{r}$ may not be metric, describing the image is straightforward:

Proposition 3.17 Suppose $\iota: A_{s} \hookrightarrow B_{r}$ is an embedding which is not metric. Then the image $\iota\left(A_{s}\right)$ has a splint $\left(s A_{1}, A_{s-1}\right)$ where $s A_{1}$ consists of short roots of $B_{r}$ and $A_{s-1} \hookrightarrow B_{r}$ is a metric embedding.

\section{ProOF.}

If the embedding is not metric, then at least one of the roots is short. Since there is only one orbit of roots under the action of the Weyl group for $A_{s}$, one may assume without loss of generality that one of the simple roots on one of the ends of its Dynkin diagram is short. In this case, one obtains exactly $s$ short roots. These roots comprise an orthogonal set and the balance is embedded metrically.

Proposition 3.18 The root system $B_{4}$ has exactly one splint.

\section{Proof.}

Suppose $\left(\Delta_{1}, \Delta_{2}\right)$ is a splint of $B_{4}$. Since $\left|B_{4}\right|=16$, one may assume without loss of generality that $8 \leq\left|\Delta_{1}\right|<16$. Examining all the root systems with rank less than or equal to 4 and with these cardinalities, one sees that $\Delta_{1}$ must be isomorphic to one of $B_{3}, A_{1}+B_{3}$, $C_{3}, A_{1}+C_{3}, 2 B_{2}$, or $D_{4}$. One must rule out all but one of these possibilities.

It is possible that $\Delta_{1}$ is isomorphic to $D_{4}$. However, in this case, the embedding $D_{4} \hookrightarrow B_{4}$ is metric and therefore is the splint $\left(D_{4}, 4 A_{1}\right)$ into long and short roots. Next, $\Delta_{1}$ cannot be $C_{3}$ or $A_{1}+C_{3}$ because $C_{3}$ does not embed in $B_{r}$ for any value of $r$.

Suppose one of the simple components of the splint is $B_{3}$. Then the embedding is metric and one may assume that the image is

$$
\left\{e_{1}, e_{2}, e_{3}, e_{1} \pm e_{2}, e_{1} \pm e_{3}, e_{2} \pm e_{3}\right\} .
$$

One can check that $A_{2}$ does not embed in the set of 7 roots not lying in this image, and hence that $B_{3}$ cannot be a component.

Finally, suppose $\Delta_{1} \cong 2 B_{2}$. Then $\left|\Delta_{1}\right|=8$ and hence $\left|\Delta_{2}\right|=8$. However, there is only one root system with rank less than or equal to 4 and with cardinality 8 , namely $2 B_{2}$. Since $\Delta_{1} \cong 2 B_{2}$, each component is metric and hence $\Delta_{1}$ contains all 4 short roots of $B_{4}$. It is not possible that $\Delta_{2}$ also contain all 4 short roots of $B_{4}$, so $\Delta_{1}$ cannot be isomorphic to $2 B_{2}$. 
Proposition 3.19 If $r \geq 5$, then $B_{r}$ has exactly one splint.

\section{ProOF.}

Suppose $r \geq 5$ and $\left(\Delta_{1}, \Delta_{2}\right)$ is a splint of $B_{r}$ other than $\left(D_{r}, r A_{1}\right)$. Consider restricting this splint to the long roots, that is, to $D_{r} \hookrightarrow B_{r}$. Notice that both $\Delta_{1}$ and $\Delta_{2}$ must each contain at least one long root of $B_{r}$, so the restriction is not trivial. If $\Delta$ is any simple component of the splint which is not embedded metrically, then $\Delta=A_{s}$ for some $s$. However, in this case, the restriction to the long roots is a metric embedding. Thus, restriction yields a splint of $D_{r}$ with $r \geq 5$, a contradiction.

\section{$C_{r}$}

If $r \geq 3$, then there is a splint $\left(r A_{1}, D_{r}\right)$ given as

$$
\Delta_{1}=\left\{2 e_{i}: 1 \leq i \leq r\right\} \text {, and } \Delta_{2}=\left\{e_{i} \pm e_{j}: 1 \leq i<j \leq r\right\} .
$$

This splint is distinguished because both components are embedded metrically. The root system $C_{3}$ has an additional splint $\left(A_{1}+B_{2}, A_{1}+A_{2}\right)$, which may be presented as

$$
\Delta_{1}=\left\{e_{2}-e_{3}\right\} \cup\left\{2 e_{1}, 2 e_{2}, e_{1} \pm e_{2}\right\}, \text { and } \Delta_{2}=\left\{e_{2}+e_{3}\right\} \cup\left\{2 e_{3}, e_{1} \pm e_{3}\right\} .
$$

Evidently, the general conclusion for the case of $C_{r}$ is similar to the case of $B_{r}$. Despite the similarity in the conclusions, however, the proofs differ considerably. The first part of the proof is to specify which simple components may appear in any splint of $C_{r}$. Obviously $A_{s}, C_{s}$, and $D_{s}$ may imbed in $C_{r}$ for certain values of $s$, whereas if $r \geq 4$, then none of the exceptional root systems may imbed in $C_{r}$. One potentiality remains:

Proposition 3.20 If $s \geq 3$, then $B_{s}$ does not imbed in $C_{r}$.

\section{Proof.}

It suffices to prove the case for $s=3$, since $B_{3}$ embeds in $B_{s}$ for all $s \geq 3$. First, if $\alpha, \beta$, and $\frac{1}{2}(\alpha+\beta)$ are roots in $C_{r}$, then $\alpha$ and $\beta$ are long and orthogonal to each other, while $\frac{1}{2}(\alpha+\beta)$ is short. Next, $B_{3}$ contains distinct roots $\left\{\alpha_{1}, \alpha_{2}, \beta_{1}, \beta_{2}\right\}$ with the property that the vector

$$
\frac{\alpha_{1}+\beta_{1}}{2}=\frac{\alpha_{2}+\beta_{2}}{2}
$$

is also a root. Suppose such roots lie in $C_{r}$. Then one immediately has

$$
\left\langle\alpha_{1}, \beta_{1}\right\rangle=\left\langle\alpha_{2}, \beta_{2}\right\rangle=0 .
$$

One can show that the inner products among the other roots are also zero as follows: Consider the value $\left\langle\alpha_{1}, \alpha_{2}\right\rangle \in\{ \pm 4,0\}$. One knows $\left\langle\alpha_{1}, \alpha_{2}\right\rangle \neq 4$ because these roots are not equal. One knows $\left\langle\alpha_{1}, \alpha_{2}\right\rangle \neq-4$ because these roots are both positive. Thus, $\left\langle\alpha_{1}, \alpha_{2}\right\rangle=0$. A similar argument holds for the inner products $\left\langle\alpha_{1}, \beta_{2}\right\rangle,\left\langle\alpha_{2}, \beta_{1}\right\rangle$, and $\left\langle\beta_{1}, \beta_{2}\right\rangle$. However, this implies that the "central" root $\frac{1}{2}\left(\alpha_{1}+\beta_{2}\right)$ has norm zero, a contradiction. 
Proposition 3.21 If $s \geq 4$, then every embedding $D_{s} \hookrightarrow C_{r}$ is metric.

\section{ProOF.}

Consider the analysis of the $F_{4}$ root system. Recall that one has a metric embedding $C_{4} \hookrightarrow F_{4}$. However, every non-metric embedding $D_{4} \hookrightarrow F_{4}$ contains 3 long roots, two having the form $e_{1} \pm e_{i}+e_{j}+e_{k}$, and the other having the form $2 e_{i}$. Evidently this does not restrict to a non-metric embedding $D_{4} \hookrightarrow C_{4}$, so this establishes the case $s=4$. If $s>4$, then every embedding $D_{s} \hookrightarrow C_{r}$ must restrict to a metric embedding $D_{4} \hookrightarrow C_{r}$. Hence the embedding of $D_{s}$ is metric.

Lemma 3.22 If $r \geq 4$ and $\left(\Delta_{1}, \Delta_{2}\right)$ is a splint of $C_{r}$ where every simple component is embedded metrically, then it is the splint $\left(r A_{1}, D_{r}\right)$.

\section{ProOF.}

This is routine to establish if $r=4$. Next, suppose $r \geq 5$. Let $\iota: D_{r} \hookrightarrow C_{r}$ be the unique metric embedding, and restrict the splint to the image of $\iota$. It was shown earlier that $D_{r}$ does not splinter when $r \geq 5$, so one may assume without loss of generality that $\Delta_{2}$ contains all the short roots of $C_{r}$. Since $\Delta_{2}$ contains all the short roots of $C_{r}$ while it is not equal to $C_{r}$, it must be simple and therefore isomorphic to $D_{r}$.

It is important to understand the contrapositive of this lemma: If $\left(\Delta_{1}, \Delta_{2}\right)$ is a splint of $C_{r}$ for which there exists $s \geq 2$ such that $A_{s}$ is a metrically embedded component in either $\Delta_{1}$ or $\Delta_{2}$, then at least one simple component of either $\Delta_{1}$ or $\Delta_{2}$ is embedded nonmetrically. Moreover, under such circumstances, such a component is isomorphic to $A_{t}$, embedded non-metrically, for some $t \geq 2$.

The rest of the proof relies on techniques similar to those used for the cases of $A_{r}, D_{r}$, and $B_{r}$. However, this case is different because of the potential occurrence of a non-metrically embedded copy of $A_{s}$. Fortunately, it is easy to describe a non-metric embedding $A_{s} \hookrightarrow C_{r}$ :

Lemma 3.23 Suppose $\iota: A_{s} \hookrightarrow C_{r}$ is a non-metric embedding with $s \geq 2$. Then (a) there is a unique index $i$ such that $2 e_{i}$ lies in the image of $\iota$, and (b) there is a subset $J \subset\{1,2,3, \ldots, r\}$ having the property that $e_{i}+e_{j}$ is in the image of $\iota$ if and only if $j \in J$.

\section{ProOF.}

First, if such an embedding contains no long roots, then it restricts to an embedding $A_{s} \hookrightarrow D_{r}$. Every such embedding is necessarily metric, so the non-metric embedding $A_{s} \hookrightarrow$ $C_{r}$ contains at least one long root, say $2 e_{i}$. Notice that the root system $A_{r}$ contains only one orbit of roots under the action of the corresponding Weyl group, so, without loss of generality, one may assume that $2 e_{i}$ is the simple root corresponding to one of the ends of 
the Dynkin diagram of $A_{s}$. If $2 e_{i}$ is a simple root, then all the other simple roots must be short. This is due to the fact that the coefficients must sum to either 0 or 2 . Were $2 e_{j}$ another simple root, there would be a root in $C_{r}$ whose coordinate sum totaled 4 . One can check that the remaining roots must also be short. Part (b) follows immediately.

It is convenient to refer to the set $J$ as the "distinguished indices" corresponding to the embedding $\iota: A_{s} \hookrightarrow A_{r}$. The corollary follows immediately:

Corollary 3.24 Suppose $\iota: A_{s} \hookrightarrow C_{r}$ is a non-metric embedding with $s \geq 2,2 e_{i}$ lies in the image of $\iota$, and $J \subset\{1,2,3, \ldots, r\}$ is the set of distinguished indices. If $j, k \in J$ with $j<k$ and neither $j$ nor $k$ is equal to $i$, then either $e_{j}-e_{k}$ or $e_{j}+e_{k}$ (exclusively) is in the image of $\iota$.

Lemma 3.25 Suppose $\Delta$ is a simple root system and that $\iota: C_{s} \hookrightarrow C_{r}$ and $\kappa: \Delta \hookrightarrow C_{r}$ are embeddings. Then the intersection $\iota\left(C_{s}\right) \cap \kappa(\Delta)$ is a root system.

\section{Proof.}

First, $\iota$ is necessarily a metric embedding. Thus, if $\kappa$ is metric, then the result follows immediately. The only non-trivial case occurs if $\Delta$ is $A_{t}$ for some $t$ and $\kappa$ is non-metric. Thus, suppose $\Delta=A_{t}$ for some $t$ and $\kappa$ is non-metric. The form of the roots in the image of $\kappa$ was described in an earlier proposition. Thus, assume $2 e_{i}$ is the unique long root appearing in the image of $\kappa$ and $I \subset\{1,2,3, \ldots, r\}$ is the set of distinguished indices. For each element $j \in\{1,2,3, \ldots, r\}$, the restriction to the orthogonal complement of $2 e_{j}$ yields a restriction of $A_{s}$ to $C_{r-1}$. Consider the following three cases: First, $\left(2 e_{i}\right)^{\perp} \cap \kappa\left(A_{t}\right)$ is a metric embedding of $A_{t-2}$ in $C_{r-1}$. Second, if $j \in I$ and $j \neq i$, then $\left(2 e_{j}\right)^{\perp} \cap \kappa\left(A_{t}\right)$ is a non-metric embedding of $A_{t-1}$. Third, if $j \notin I$, then $\left(2 e_{j}\right)^{\perp} \cap \kappa\left(A_{t}\right)=\kappa\left(A_{t}\right)$, for none of the indices in $\kappa\left(A_{t}\right)$ are equal to $j$.

For the general case, notice that there is a subset $J \subset\{1,2,3, \ldots, r\}$ such that $2 e_{i}$ lies in the image of $\iota$ if and only if $i \in J$. One may then apply the above arguments inductively, starting with $t=r-1$ and proceeding down until $t=|J|$.

Corollary 3.26 Suppose $\left(\Delta_{1}, \Delta_{2}\right)$ is a splint of $C_{r}$ and $\iota: C_{s} \hookrightarrow C_{r}$ is an embedding. Then the restriction of $\left(\Delta_{1}, \Delta_{2}\right)$ to the image of $\iota$ is a splint of $C_{s}$.

Proposition 3.27 If $r \geq 4$, then $C_{r}$ has exactly one splint.

\section{ProOF.}

Suppose $\left(\Delta_{1}, \Delta_{2}\right)$ is a splint of $C_{r}$ which is not equivalent to $\left(r A_{1}, D_{r}\right)$. Then at least one simple component $\Delta$ is embedded non-metrically. This component $\Delta$ cannot be $D_{s}$ 
or $C_{s}$ for each of these must embed metrically. Nor can $\Delta$ be $B_{s}$ or any exceptional root system for none of these embed in $C_{r}$. The only remaining possibility is that $\Delta \cong A_{s}$ for some $s \geq 2$. This immediately leads to a contradiction because then this splint restricts to a splint of $C_{4}$ having $A_{2}$ as a component.

\section{Conclusion}

This classification has not addressed the issue of equivalence. Indeed, one may define: If $\Delta$ is a simple root system with Weyl group $W$, then the splints $\left(\Delta_{1}, \Delta_{2}\right)$ and $\left(\Delta_{1}^{\prime}, \Delta_{2}^{\prime}\right)$ of $\Delta$ are "equivalent" if there exists $\sigma \in W$ such that

$$
\sigma \cdot\left(\Delta_{1} \cup\left(-\Delta_{1}\right), \Delta_{2} \cup\left(-\Delta_{2}\right)\right)=\left(\Delta_{1}^{\prime} \cup\left(-\Delta_{1}^{\prime}\right), \Delta_{2}^{\prime} \cup\left(-\Delta_{2}^{\prime}\right)\right) .
$$

The general problem is to detect whether or not two splints are equivalent. As the reader must have noticed by now, however, splints of simple root systems are scarce and easy to describe, so it would not take much effort to determine that the table is complete up to equivalence. The splints of $A_{4}$ and $D_{4}$ probably afford the most non-trivial (and most interesting) cases.

There may be a more efficient way to achieve the classification of splints of simple root systems than that given here, although this author has not found one.

\section{Appendix}

Each row in the following table gives a splint $\left(\Delta_{1}, \Delta_{2}\right)$ of the simple root system $\Delta$.

\begin{tabular}{cc||c|c} 
type & $\Delta$ & $\Delta_{1}$ & $\Delta_{2}$ \\
\hline \hline (i) & $G_{2}$ & $A_{2}$ & $A_{2}$ \\
& $F_{4}$ & $D_{4}$ & $D_{4}$ \\
\hline (ii) & $B_{r}(r \geq 2)$ & $D_{r}$ & $r A_{1}$ \\
& $C_{r}(r \geq 3)$ & $r A_{1}$ & $D_{r}$ \\
\hline (iii) & $A_{r}(r \geq 2)$ & $A_{r-1}$ & $r A_{1}$ \\
& $B_{2}$ & $A_{1}$ & $A_{2}$ \\
\hline (iv) & $B_{2}$ & $2 A_{1}$ & $2 A_{1}$ \\
& $A_{3}$ & $A_{1}+D_{2}$ & $A_{1}+D_{2}$ \\
& $A_{4}$ & $A_{2}+D_{2}$ & $A_{2}+D_{2}$ \\
& $D_{4}$ & $2 A_{2}$ & $2 A_{2}$ \\
\hline (v) & $A_{4}$ & $2 A_{2}$ & $2 D_{2}$ \\
& $A_{r}(r \geq 3)$ & $(r-1) A_{1}$ & $A_{1}+A_{r-1}$ \\
\hline (vi) & $B_{3}$ & $3 A_{1}$ & $A_{3}$ \\
& $C_{3}$ & $A_{1}+B_{2}$ & $A_{1}+A_{2}$ \\
& $G_{2}$ & $2 A_{1}$ & $B_{2}$
\end{tabular}


This table is organized into several types: (i) Both $\Delta_{1}$ and $\Delta_{2}$ are embedded metrically, and $\Delta_{1} \cong \Delta_{2}$. (ii) Both $\Delta_{1}$ and $\Delta_{2}$ are embedded metrically, but $\Delta_{1}$ and $\Delta_{2}$ are not isomorphic. (iii) Only $\Delta_{1}$ is embedded metrically; (iv) Every simple component is embedded metrically and $\Delta_{1} \cong \Delta_{2}$. (v) Every simple component is embedded metrically, but $\Delta_{1}$ and $\Delta_{2}$ are not isomorphic. (vi) At least one simple component of $\Delta_{2}$ is not embedded metrically. The notation $D_{2}$ is used in a few instances to designate a metric embedding of $2 A_{1}$. The types (i), (ii), and (iii) share the property that $\Delta_{1}$ corresponds to a Lie subalgebra of the Lie algebra with root system $\Delta$.

\section{References}

[1] Sara Billey and Alexander Postnikov. Smoothness in Schubert varieties via patterns in root subsystems. Adv. Appl. Math. 34 (2005), 447-466.

[2] G. J. Heckman. Projections of orbits and asymptotic behavior of multiplicities for compact connected Lie groups. Invent. Math. 67 (1982), no.2, 333-356.

[3] James E. Humphreys. Reflection Groups and Coxeter Groups. Cambridge University Press, 1992.

[4] Nathan Jacobson. Lie Algebras. Dover Publications Inc., New York, 1979.

[5] King, R. C. and Qubanchi, A. H. A. The evaluation of weight multiplicities of $G_{2} . J$. Phys. A 11 (1978), no. 8, 1491-1499.

[6] V. D. Lyakhovsky, S. Yu Melnikov, et al. Recursion relations and branching rules for simple Lie algebras. J. Phys. A: Math. Gen. 29 (1996) 1075-1087. 